\title{
USING GLOBAL INTERPOLATION TO EVALUATE THE BIOT-SAVART INTEGRAL FOR DEFORMABLE ELLIPTICAL GAUSSIAN VORTEX ELEMENTS
}

\author{
RODRIGO B. PLATTE*, LOUIS F. ROSSI ${ }^{\dagger}$, AND TRAVIS B. MITCHELL ${ }^{\ddagger}$
}

\begin{abstract}
.
This paper introduces a new method for approximating the Biot-Savart integral for elliptical Gaussian functions using high-order interpolation and compares it to an existing method based on small aspect ratio asymptotics. The new evaluation technique uses polynomials to approximate the kernel corresponding to the integral representation of the streamfunction. We determine the polynomial coefficients by interpolating precomputed values from look-up tables over a wide range of aspect ratios. When implemented in a full nonlinear vortex method, we find that the new technique is almost three times faster and unlike the asymptotic method, provides uniform accuracy over the full range of aspect ratios. As a proof-of-concept for large scale computations, we use the new technique to calculate inviscid axisymmetrization and filamentation of a two-dimensional elliptical fluid vortex. We compare our results with those from a pseudo-spectral computation and from electron vortex experiments, and find good agreement between the three approaches.
\end{abstract}

Key words. Vortex methods, Navier-Stokes equations, Biot-Savart integral, high Reynolds number flow, electron vortices.

1. Introduction. This paper introduces a new scheme for calculating the BiotSavart integral of an elliptical Gaussian distribution and compares it to an existing, asymptotic method. The immediate application of these techniques is to rapidly determine the streamfunction and associated derivatives of an elliptical Gaussian vorticity distribution and for a fourth-order viscous core-spreading vortex method. The mathematical problem, concisely stated, is to find the streamfunction $\psi$ where

$$
\begin{gathered}
\psi(\vec{x} ; \sigma, a)=-\frac{1}{4 \pi} \iint_{-\infty}^{\infty} \log \left(|\vec{x}-\vec{s}|^{2}\right) \phi(\vec{s}) d s_{1} d s_{2}, \\
\phi(\vec{x} ; \sigma, a)=\frac{1}{4 \pi \sigma^{2}} \exp \left[-\frac{\left(x^{2} / a^{2}+y^{2} a^{2}\right)}{4 \sigma^{2}}\right]
\end{gathered}
$$

where $\phi$ is a function describing the shape of the basis function, $s_{1}$ and $s_{2}$ are the components of $\vec{s}$, and $\sigma^{2}$ and $a^{2}$ is the core size and aspect ratio, respectively, of the basis function. Equation (1.1a) is a restatement of the Biot-Savart integral for the velocity field restricted to a two-dimensional flow:

$$
\vec{u}=\left[\begin{array}{r}
\frac{\partial \psi}{\partial y} \\
-\frac{\partial \psi}{\partial x}
\end{array}\right]=-\frac{1}{2 \pi} \iint_{-\infty}^{\infty} \frac{1}{|\vec{x}-\vec{s}|^{2}} R(\vec{x}-\vec{s}) \phi(\vec{s}) d \vec{s}
$$

where $R$ is a rotation $R[x, y]^{T}=[y,-x]^{T}$. We capture translations and rotations through symmetries in (1.1a) and apply them to the problem during pre- and posttreatment of the evaluation. An earlier paper [35] describes an asymptotic approach built on top of Lamb's exact solution for an elliptical patch of vorticity.

\footnotetext{
*Oxford University Computing Laboratory, Wolfson Bldg., Parks Road, Oxford, OX1 3QD, United Kingdom (rodp@comlab.ox.ac.uk). After December 2009: Arizona State University, Department of Mathematics and Statistics, Tempe, AZ, 85287-1804.

${ }^{\dagger}$ Department of Mathematical Sciences, University of Delaware, Newark DE 19716

$\ddagger 103$ Cornell Ave., Swarthmore PA 19081
} 
This is an important problem for two reasons. First, small numbers of elliptical Gaussian vortices may be useful for low order models (see [32, 38, 39, 44] for examples of this approach). Second, elliptical Gaussian basis functions are the foundation of a new class of fourth-order vortex methods for solving the viscous Navier Stokes equations. The use of rigid and deforming elliptical vortex patches dates back to efforts in the 1980s and early 1990s by Teng and his co- authors as a means of improving accuracy by adapting to asymmetric flow geometries [40, 41, 42]. In their later work, patches would deform by following the nearby flow geometry. Meiburg applied a similar approach to simulate shear layers [21], and Ojima and Kamemoto use a hybrid deforming element in a three dimensional vortex code [29]. Deforming elliptical Gaussians capture both linear convection and diffusion in two-dimensions and so they are a natural choice for a high spatial order method. Following this line of reasoning, Moeleker and Leonard performed a series of computational experiments using deforming elliptical Gaussian basis functions for linearized convection-diffusion equations but without the expected increase in spatial accuracy [16, 25]. Shortly afterward, Rossi found an additional requirement for this boost in accuracy that can be satisfied when curvature corrections are applied to the velocity field, and demonstrated that methods using deforming blobs will outperform methods using rigid blobs even at moderate problem sizes $[33,34]$.

With this issue resolved, a small number of mathematical problems barred the way to a full-fledged high order viscous vortex method. One of them is a fast, effective means of evaluating the streamfunction and its first three derivatives for the elliptical Gaussian as described in (1.1). The basis functions are deformed by local flow deviations, and the velocity field correction requires that one calculate the velocity curvature (see Appendix A for an overview of the dynamical system), so calculating the streamfunction and its derivatives is critical to an effective computation. While there are a variety of methods that can be applied for integrating axisymmetric distributions due to the obvious reduction in dimensionality, the non-axisymmetric problem is more general and challenging. Rossi proposed an accurate and effective means of solving this problem, verified the full nonlinear convergence of the vortex method and developed a fast summation algorithm for elliptical Gaussians in the far field [35]. In this paper, we focus on the direct evaluation of elliptical Gaussians in the near field, and we present a significant improvement over this earlier solution.

This manuscript, describing a new, more accurate and more efficient global method for solving (1.1), is organized as follows. This section of the paper describes the problem in its broader context. We briefly review the asymptotic method for evaluation of the streamfunction in Section 2. Section 3 describes the new technique for evaluating the streamfunction. Section 4 quantitatively compares the existing asymptotic method and the new evaluation technique to one another. We demonstrate full nonlinear converence to the exact solution of the Navier-Stokes equation for the test problem. Section 5 uses the new method to compute a challenging inviscid filamentation problem as a proof-of-concept, and we compare our results with a standard pseudo-spectral method and electron vortex experiments [23]. Our results and findings will be summarized in Section 6 .

2. Review of asymptotic method. Both the new technique and the asymptotic method introduced in [35] rely upon Lamb's expression for the streamfunction 
of an elliptical patch with axes $l_{1}$ and $l_{2}$ [13].

$$
\begin{aligned}
& \psi= \begin{cases}\frac{1}{2 \pi\left(l_{1}+l_{2}\right)}\left(\frac{x^{2}}{l_{1}}+\frac{y^{2}}{l_{2}}\right), & (x, y) \in \mathcal{E}\left(l_{1}, l_{2}\right) \\
\frac{1}{2 \pi}\left[\ln \left(\frac{\alpha+\beta}{l_{1}+l_{2}}\right)+\frac{\frac{x^{2}}{\alpha}+\frac{y^{2}}{\beta}}{\alpha+\beta}\right], & (x, y) \notin \mathcal{E}\left(l_{1}, l_{2}\right)\end{cases} \\
& \alpha=\sqrt{l_{1}^{2}+\xi}, \\
& \beta=\sqrt{l_{2}^{2}+\xi}, \\
& 1=\left(\frac{x^{2}}{l_{1}^{2}+\xi}\right)+\left(\frac{y^{2}}{l_{2}^{2}+\xi}\right),
\end{aligned}
$$

where $\mathcal{E}\left(l_{1}, l_{2}\right)$ is the support of the ellipse and

$$
\vec{u}=\left[\begin{array}{l}
u \\
v
\end{array}\right]=\left[\begin{array}{c}
-\frac{\partial \psi}{\partial y} \\
\frac{\partial \psi}{\partial x}
\end{array}\right] .
$$

Following the procedure in [35], we can express the streamfunction of an elliptical Gaussian at a point $\left(x_{*}, y_{*}\right)$ as

$$
\begin{aligned}
\psi\left(x_{*}, y_{*}\right) & =-\int_{0}^{R_{*}} \psi_{1} \partial_{R} \phi R^{2} d R-\int_{R_{*}}^{\infty} \psi_{2} \partial_{R} \phi d R, \\
\phi(R ; \sigma, a) & =\frac{1}{4 \pi \sigma^{2}} e^{-R^{2} / 4 \sigma^{2}} . \\
\psi_{1} & =\frac{1}{2}\left[\frac{\left(\frac{x_{*}{ }^{2}}{\alpha}+\frac{y_{*}{ }^{2}}{\beta}\right)}{\alpha+\beta}+\ln \left(\frac{\alpha+\beta}{R a+R / a}\right)\right], \\
\psi_{2} & =\frac{1}{2} \frac{\frac{x_{*}^{2}}{a}+y_{*}^{2} a}{a+1 / a} \\
\alpha & =\sqrt{R^{2} a^{2}+\xi} \\
\beta & =\sqrt{\frac{R^{2}}{a^{2}}+\xi} .
\end{aligned}
$$

where $\rho_{*}{ }^{2}=x_{*}{ }^{2}+y_{*}{ }^{2}, R_{*}{ }^{2}=\frac{x_{*}{ }^{2}}{a^{2}}+y_{*}{ }^{2} a^{2}$ and

$$
\xi=\frac{1}{2}\left\{\rho_{*}{ }^{2}-R^{2}\left(a^{2}+\frac{1}{a^{2}}\right)+\sqrt{\left[R^{2}\left(a^{2}+\frac{1}{a^{2}}\right)-\rho_{*}{ }^{2}\right]^{2}+4 R^{2}\left(R_{*}{ }^{2}-R^{2}\right)}\right\} .
$$

The second integral poses no difficulty at all because $\psi_{2}$ is not a function of $R$. However, there is no known expression for the first integral in terms of elementary functions, and this is where the challenge lies.

The asymptotic method approximates $\psi_{1}$ in powers of the small parameter

$$
\epsilon=\frac{a-1}{a+1}
$$

which reduces the first part of the integral in (2.2a) to moments of a one-dimensional Gaussian. The coefficients of each of the moments depend upon $x_{*}, y_{*}, a$ and $\sigma$. 
Successive moments can be obtained through a recurrence relation, so the method is both fast and accurate. Unfortunately, it requires many terms in the large aspect ratio limit, so the evaluation of the moments becomes unstable at high orders due to catastrophic cancellations.

3. The high-order interpolation method with domain decomposition. The asymptotic approximation given in $\S 2$ converges most rapidly in the small $\epsilon$ limit and so is most accurate when vorticity blobs are nearly isotropic. On the other hand, Marshall and Grant derived an approximation for the velocity field when blobs are highly anisotropic in [18]. In this section we explore a high-order interpolation technique that uses domain decomposition for the fast evaluation of the Biot-Savart integral for all core aspect ratios - although we restrict our implementation to $a \in[0.1,10]$, since larger aspect ratios would be better handled by the closed form expressions derived in [18].

Without loss of generality, in this section, we assume $\sigma \equiv 1$ and use the notation $\psi(\vec{x} ; a) \equiv \psi(\vec{x} ; 1, a)$. Notice that

$$
\psi(\vec{x} ; \sigma, a)=\frac{1}{\sigma} \psi(\vec{x} / \sigma ; a)+C_{\sigma, a}
$$

where $C_{\sigma, a}$ does not depend on $\vec{x}$ and does not affect the velocity field; we set $C_{\sigma, a} \equiv 0$ in our implementation. Moreover, we restrict our computations to $a \geq 1, x>0$, and $y>0$, since

$$
\psi(x, y ; 1 / a)=\psi(y, x ; a) \quad \text { and } \quad \psi(y, x ; a)=\psi(|y|,|x| ; a) .
$$

The main idea is to recast the parameter $a$ as a variable. Because the Biot-Savart integral is a smooth function of $\vec{x}$, accurate approximations of $\psi$ can be obtained with the polynomial expansion,

$$
\psi(x, y ; a)=\sum_{n_{x}=0}^{N_{x}} \sum_{n_{y}=0}^{N_{y}} \lambda_{n_{x}, n_{y}}(a) x^{n_{x}} y^{n_{y}}, \quad x>0, y>0, a>1
$$

The coefficients $\lambda_{n_{x}, n_{y}}$ are functions of the core aspect ratio in contrast to the asymptotic method where the coefficients depend upon $x, y$ and $\epsilon$ (or $a$ ). These coefficients are computed for several values of $a$, and for each given aspect ratio, we compute them so that (3.1) interpolates the values of $\psi$ computed using (2.2a). These coefficients were obtained only once and stored in files for future use, since this operation requires time consuming computations. Because each $\lambda_{n_{x}, n_{y}}$ is a smooth function of $a$, when evaluations are needed for arbitrary values of $a$, new coefficients can be found by a fit of the coefficients already computed.

Figure 3.1 depicts the behavior of $\psi$ as a function of the three variables $x, y$, and $a$. Contours are shown for $\psi=0$ to $\psi=0.3$ in increments of 0.01 . Notice that variations of $\psi$ with respect to $a$ are more drastic for smaller values of $a$ rather than larger values. The contours in the third row of this figure show that $\psi$ has elliptic level curves near the origin, but circular for large values of $x$ and $y$, since $\psi \rightarrow c \log \left(x^{2}+y^{2}\right)$ as $x^{2}+y^{2} \rightarrow \infty$.

The number of operations required to evaluate (3.1) and its derivatives is $O\left(N_{x} N_{y}\right)$. For fast evaluations, therefore, we need $N_{x}$ and $N_{y}$ to be relatively small numbers. In order to accomplish this without compromising accuracy, we resort to domain decomposition. More specifically, we split our computational domain in 13 rectangular 

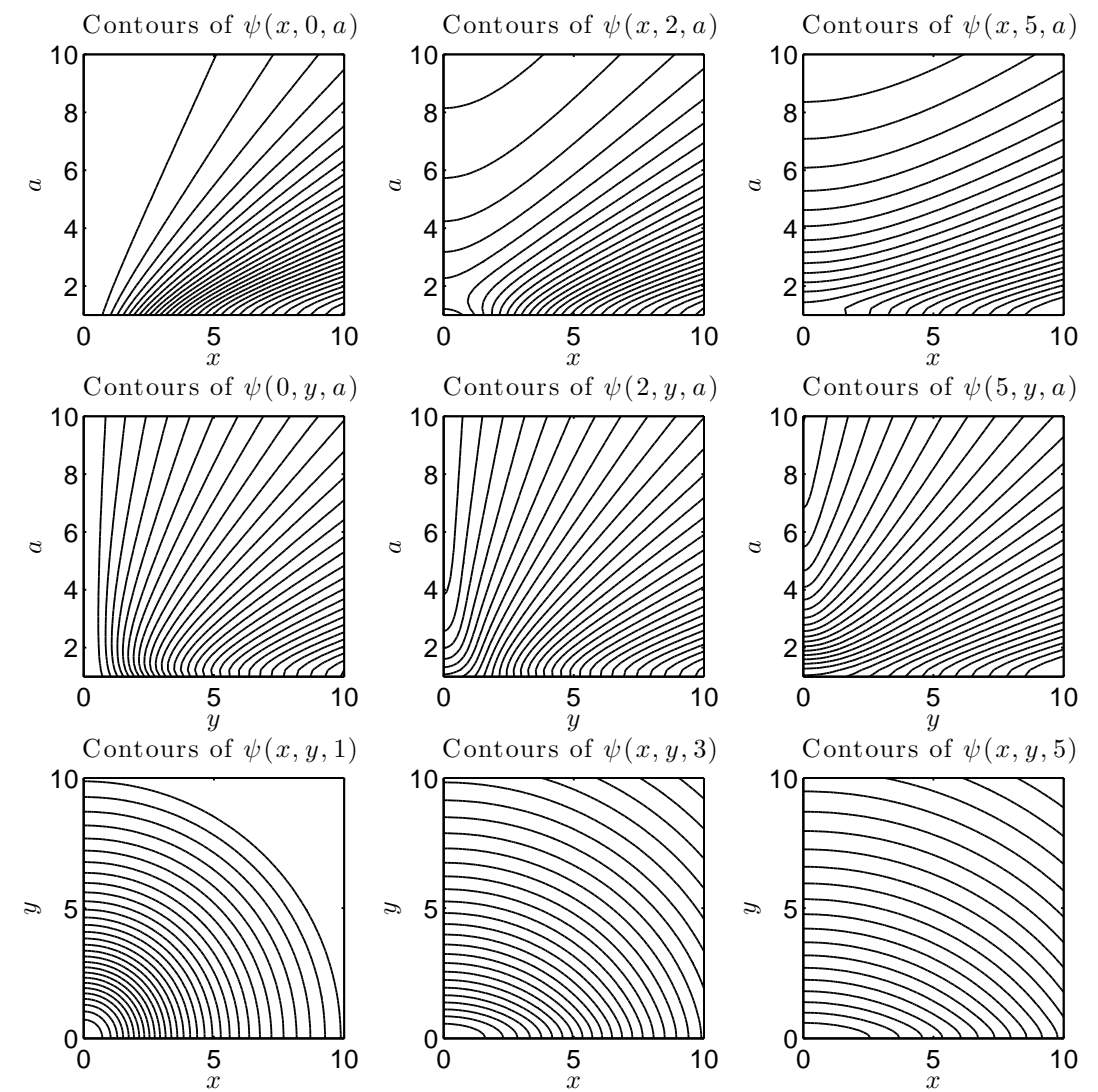

Fig. 3.1. Contour levels of $\psi$. Top row: contours for fixed values of $y$; middle row: contours for fixed values of $x$; and bottom row: contours for fixed values of $a$.

regions shown in Fig. 3.2. The exact boundary for each subdomain is presented in Table 3.1. Notice that the subdomains are scaled with $a$ since the resolution required in parts of the domain depend on the core aspect ratio. These subdomains were obtained by trial and error so that (3.1) is accurate to about 10 digits with $N x=N y=16$ in each region. For the far field approximation, $x>40.5 a$ or $y>40.5 a$, we use the isotropic approximation $\psi=c_{1} \log \left(x^{2}+y^{2}\right)$. In our implementation, therefore, we use a piecewise polynomial representation of the solution and replace (3.1) with 13 equivalent expressions each defined only on one subdomain.

It is well known that high-order polynomial interpolation is well-conditioned only on nodes that are clustered more densely near the boundaries of the domain [7]. In computing the polynomial coefficients, therefore, we mapped each domain to $[-1,1] \times$ $[-1,1]$ and used the Chebyshev points depicted in Fig. 3.3; see [43] for details. We point out that we use monomials in (3.1), rather than orthogonal polynomials, because of their fast evaluation - since the degree of the polynomials is relatively small, the interpolation process is not ill-condtioned.

The coefficients in (3.1) are computed in each individual subdomain for several 


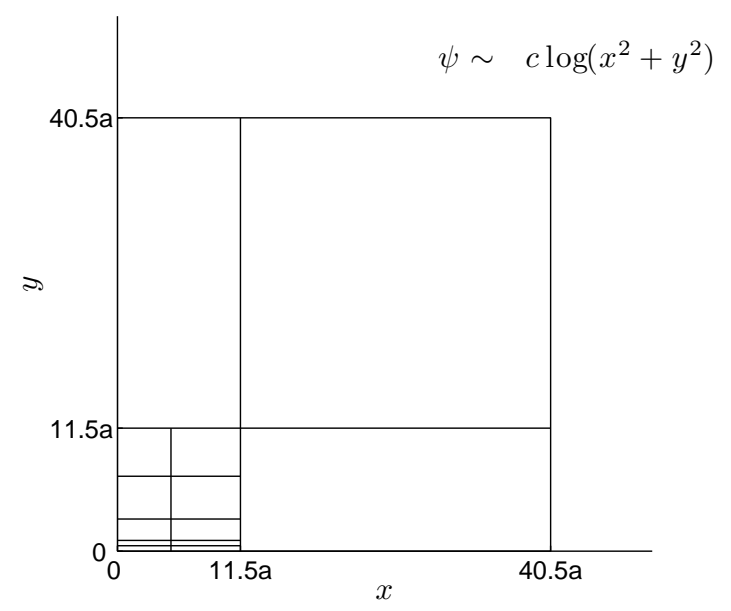

FIG. 3.2. Domain decomposition in the xy-plane.

\begin{tabular}{|c||c|c||c|c|}
\hline Subdomain & $x_{\min }$ & $x_{\max }$ & $y_{\min }$ & $y_{\max }$ \\
\hline \hline 1 & 0 & $5 a$ & 0 & 0.5 \\
2 & 0 & $5 a$ & 0.5 & 1 \\
3 & 0 & $5 a$ & 1 & 3 \\
4 & 0 & $5 a$ & 3 & $7 \sqrt{a}$ \\
5 & 0 & $5 a$ & $7 \sqrt{a}$ & $11.5 a$ \\
6 & $5 a$ & $11.5 a$ & 0 & 0.5 \\
7 & $5 a$ & $11.5 a$ & 0.5 & 1 \\
8 & $5 a$ & $11.5 a$ & 1 & 3 \\
9 & $5 a$ & $11.5 a$ & 3 & $7 \sqrt{a}$ \\
10 & $5 a$ & $11.5 a$ & $7 \sqrt{a}$ & $11.5 a$ \\
11 & $11.5 a$ & $40.5 a$ & 0 & $11.5 a$ \\
12 & 0 & $11.5 a$ & $11.5 a$ & $40.5 a$ \\
13 & $11.5 a$ & $40.5 a$ & $11.5 a$ & $40.5 a$ \\
\hline \multicolumn{5}{|c|}{ TABLE 3.1} \\
\hline
\end{tabular}

Rectangular subdomains in the $x y$-plane.

values of $a$. Because $\psi$ varies more rapidly with respect to $a$ when $a \approx 1$, we use a nonuniform distribution of values of $a$ to compute these coefficients. More precisely, we compute them for 700 aspect ratios between 1 and 10, such that the distance between two neighboring values grows linearly as $a$ increases; i.e., $a_{j}=1+9(j / 699)^{2}$, $j=0 \ldots 699$. Fig. 3.4 shows the values of $a$ used in our computations. Due to the large number of points, we only show points between 1 and 1.1. Because we have a large number of samples of coefficients, a low order approximation in the direction of the parameter $a$ yields accurate results. In fact, the errors in the approximations are in general due to the approximations in the $x$ and $y$ direction. For an arbitrary value 


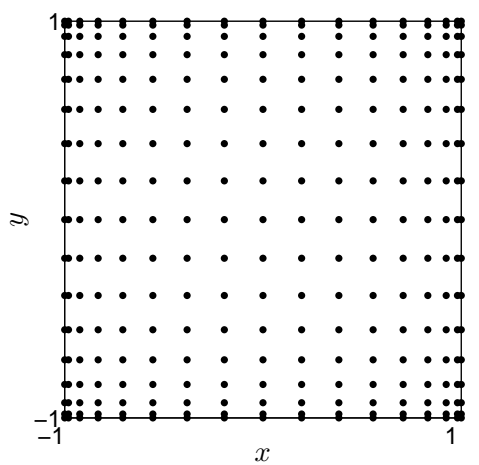

FIG. 3.3. Chebyshev grid: $17 \times 17$ points.

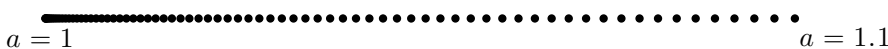

FIG. 3.4. Nonuniform sampling values of a in $[1,1.1]$. The spacing between values grows linearly.

of $a$ in $\left[a_{j}, a_{j+1}\right]$ the coefficients are computed using the weighted average,

$$
\lambda_{n_{x}, n_{y}}(a)=\frac{\left(a-a_{j}\right) \lambda_{n_{x}, n_{y}}\left(a_{j+1}\right)+\left(a_{j+1}-a\right) \lambda_{n_{x}, n_{y}}\left(a_{j}\right)}{a_{j+1}-a_{j}} .
$$

In our algorithm, the coefficients are stored in 13 files, each corresponding to a subdomain in Table 3.1. Each file stores $700 \times 17 \times 17$ double precision coefficients, requiring about $5 \mathrm{MB}$ of memory. The files are loaded only once in the beginning of an execution. The number of operations required for the evaluation of $\psi$ for an input $(x, y, a)$ is, therefore, dominated by approximately $2 \times 289$ products in $(3.2)$ and approximately $3 \times 289$ products to evaluate the polynomial in (3.1). Derivatives of $\psi$ are obtained by differentiating (3.1).

The computation of higher derivatives usually result in loss of accuracy. Although our implementation evaluates the Biot-Savart integral to an error of $O\left(10^{-10}\right)$, approximately one accurate digit is lost for each derivative computed. In order to estimate the error in the second derivatives, we use the Poisson equation

$$
\psi_{x x}+\psi_{y y}-\frac{1}{4 \pi} \exp \left[-\frac{1}{4}\left(\frac{x^{2}}{a^{2}}+y^{2} a^{2}\right)\right]=0
$$

which is solved by (1.1a). The residual in (3.3) when $a=\sqrt{5}$ is shown on the left plot of Fig. 3.5. This gray scale map corresponds to the logarithm of the residual, showing an error of $O\left(10^{-8}\right)$ on the second derivatives of $\psi$.

Similarly, we can estimate the error in the third derivatives using the equation

$$
\psi_{x x x}+\psi_{y y y}+\psi_{x y y}+\psi_{x x y}+\frac{1}{8 \pi}\left(\frac{x}{a^{2}}+y a^{2}\right) \exp \left[-\frac{1}{4}\left(\frac{x^{2}}{a^{2}}+y^{2} a^{2}\right)\right]=0,
$$

which is obtained by differentiating (3.3) with respect to $x$ and $y$ and adding the resulting equations. The residual in (3.4) is shown on the right plot of Fig. 3.5, which presents an error of $O\left(10^{-7}\right)$ near the origin for third derivatives. Similar pattern has been observed for other values of $a$. 

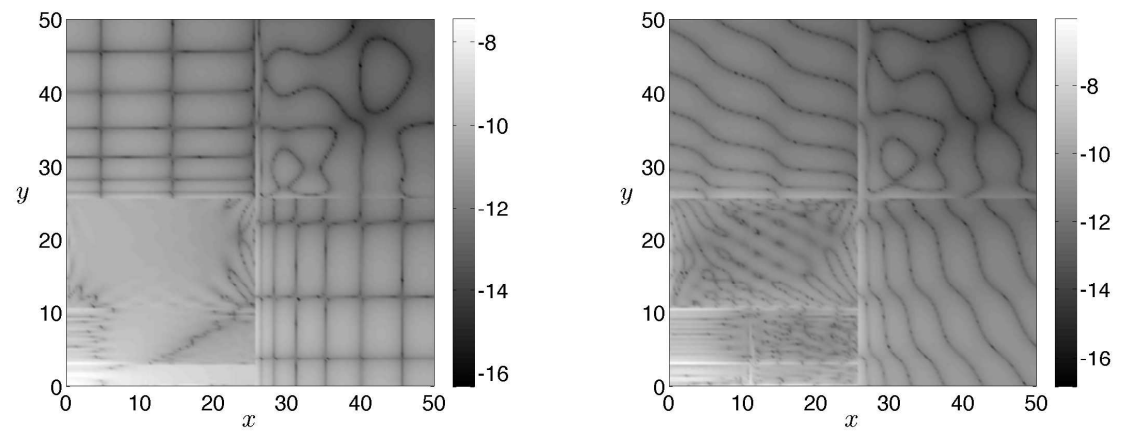

FIG. 3.5. Estimated error in second and third derivatives of $\psi$ for $a=\sqrt{5}$. Left: $\log _{10}$ of the residual in (3.3). Right: $\log _{10}$ of the residual in (3.4).

\begin{tabular}{|l|l|l|l|}
\hline Method & Far field (MP) & Near field (direct) & Velocity total \\
\hline \hline Asymptotic & 9.2 & 287.4 & 296.7 \\
\hline Spectral & 9.3 & 113.7 & 123.7 \\
\hline
\end{tabular}

Average CPU seconds dedicated to different tasks in the velocity computation over five steps through the vortex method algorithm for $N=6917$. All times are measured in $C P U$ seconds. The reported times for multipole (MP) summation include both the calculation of multipole coefficients and the summation of far field effects. The cost of the velocity computation includes lesser tasks so the total of near and far field costs do not always add up to the total.

4. Comparisons. The new evaluation method stably provides accurate estimates of the Biot-Savart integral with spectral accuracy, independent of the aspect ratio. The asymptotic method has polynomial convergence in powers of $\epsilon$ which depends upon the aspect ratio. There are some notable implementation differences. The computational simplicity of the asymptotic method allows the streamfunction coefficients to be calculated on-the-fly during a computation. The cost associated with calculating the coefficients for the spectral scheme is prohibitive, so we precomputed coefficient values for in regular increments of $a$ and then use these tables to interpolate coefficients for any value of $a$ during the computation. In these comparisons, the asymptotic coefficients are calculated as needed while the spectral coefficients are pre-calculated.

The simplest comparison is to compare CPU times for streamfunction calculations. These measurements were performed on an Intel Core 2 Duo $1.862 \mathrm{GHz} \mathrm{CPU}$ running Linux. We compiled the standalone code with GNU C compiler, gcc, version 4.1.1 with the -O3 optimization flag set. We excluded initializations and file I/O from the comparison. The test consisted of a calculation of the streamfunction as well as its first, second and third derivatives on a $100 \times 100$ mesh covering the domain $[12 \sigma, 12 \sigma]^{2}$ for an elliptical Gaussian with $\epsilon=0.25$ using a sixth order expansion with the asymptotic method and compared it to the algorithm described in $\S 3$ using $17^{2}$ coefficients. The asymptotic technique required $0.3 \mathrm{CPU}$ seconds to complete the computation whereas the spectral technique required $0.12 \mathrm{CPU}$ seconds, a reduction of almost $1 / 3$. The asymptotic method had the same accuracy reported in [35] whereas the new method was numerically indistinguishable from the high precision quadrature used as the reference. 


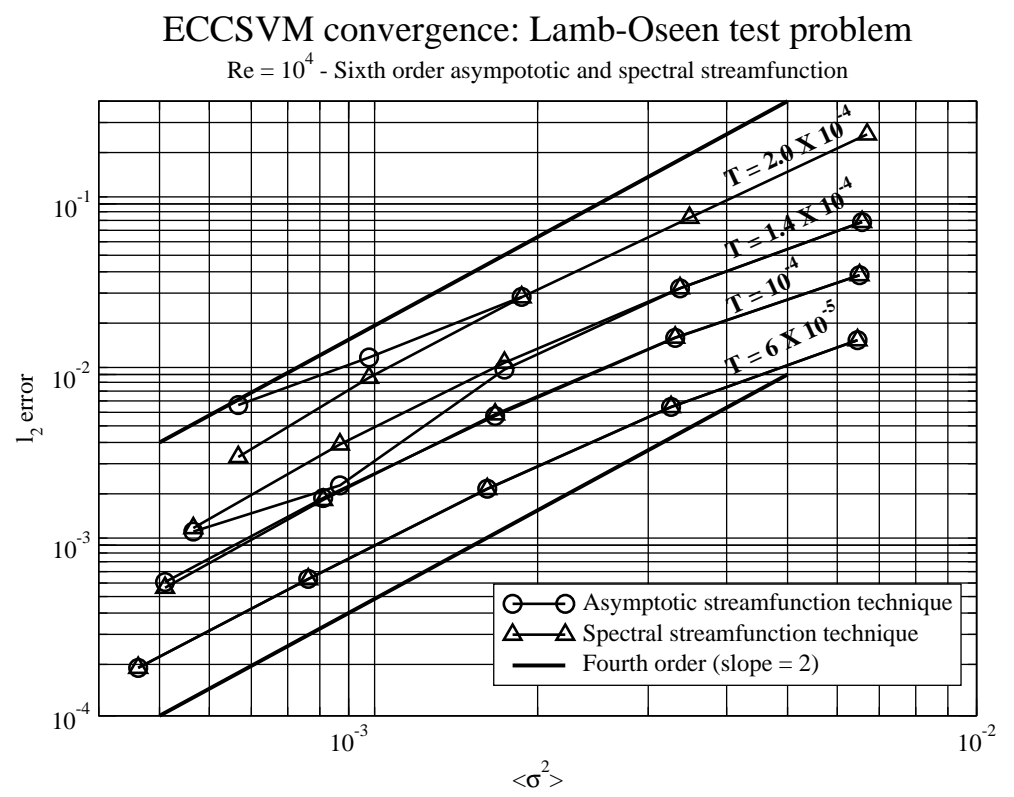

FIG. 4.1. Results from the spectral and asymptotic streamfunction evaluation at times $T=$ $6 \times 10^{-5}, 10^{-4}, 1.4 \times 10^{-4}$ and $2 \times 10^{-4}$. Only the finer three data points are available for $T=$ $2.0 \times 10^{-4}$ because the asymptotic velocity evaluations experience catastrophic cancellation errors when the aspect ratios grow large.

However, this does not paint a complete picture in practice. To be competitive, vortex calculations must include some form of fast summation that separates the near and far field. The methods discussed in this paper are limited to direct evaluations in the near field. Even for large-scale computations, the majority of effort will be expended on direct evaluations. In Table 4.1, the computational costs are broken down between near and far field. For this test, we used a test problem with $N=6917$ basis functions (corresponding to the third most refined run of five runs shown in Fig. 4.1). The improvement in performance is substantial. Tests over different problem sizes scale with $N$, and when parallelized, scale with the number of CPUs.

A standard test for a vortex code is the steady diffusion of an axisymmetric distribution of vorticity. We will use a Lamb-Oseen vortex

$$
\omega(\vec{x}, 0)=4 \exp \left(-4|\vec{x}|^{2}\right),
$$

where $\omega$ is the vorticity field. The exact solution is

$$
\omega(\vec{x}, t)=\frac{1}{\frac{1}{4}+4 t / \operatorname{Re}} \exp \left(-\frac{|\vec{x}|^{2}}{\frac{1}{4}+4 t / \operatorname{Re}}\right),
$$

where Re is the Reynolds number of the flow. This is a strong test for any method's ability to capture convective forces correctly because the streamlines are concentric. A properly implemented low-order vortex method should nail this problem to within numerical precision regardless of the core size because it ignores linear flow deviations that cross streamlines. This is a special advantage unique to flow with circular streamlines. For the fourth-order method, the Lamb-Oseen problem is not so special and spatial errors are finite and measurable. We performed a direct comparison between 


\section{Basis function aspect ratio growth}

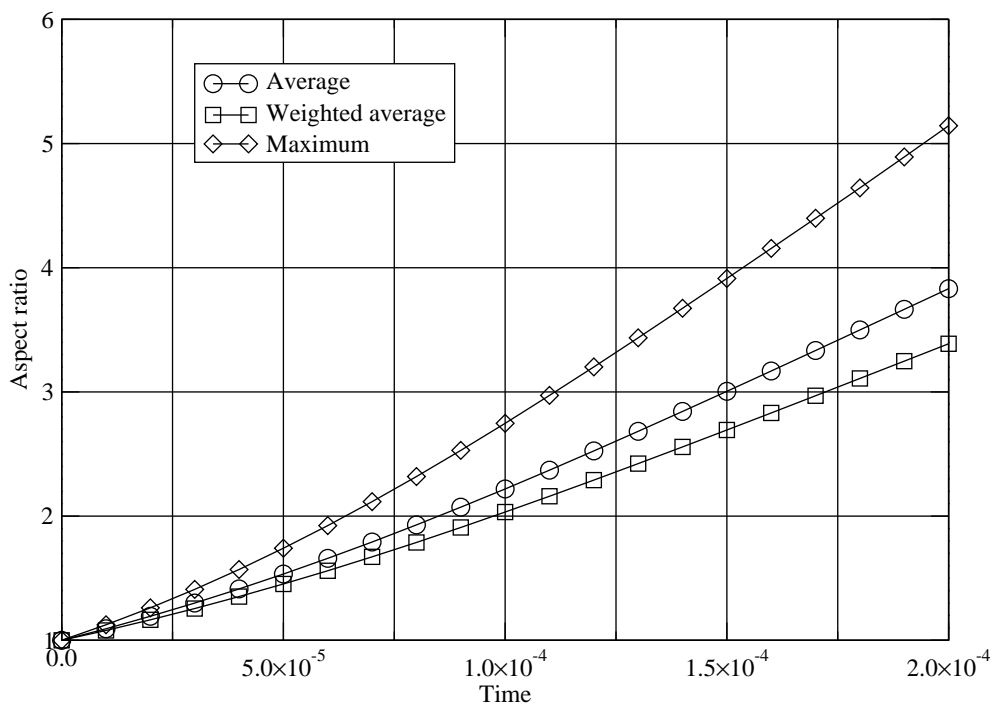

FIG. 4.2. Typical aspect ratios for the Lamb-Oseen test problem as a function of time for $\sigma^{2}=4 \times 10^{-4}$ at $t=0$ using the asymptotic technique. Initial conditions using other initial blob core sizes exhibit similar aspect ratio growth curves.

the asymptotic and spectral technique as shown in Fig. 4.1. For early evolution times, both methods produce comparable precision and exhibit fourth order accuracy. However, at later times, the basis functions become more deformed and the asymptotic streamfunction approximation becomes less precise. At $T=2.0 \times 10^{-4}$ for large $\left\langle\sigma_{i}^{2}\right\rangle$, the aspect ratio becomes so large that the sixth-order expansion becomes numerically unstable and the algorithm halts. Typical growth curves for the average aspect ratio $(1 / N) \sum_{i=1}^{N} a_{i}^{2}$, circulation weighted average aspect ratio $\left(1 / \sum_{i=1}^{N} \gamma_{i}\right) \sum_{i=1}^{N} \gamma_{i} a_{i}^{2}$ and maximum aspect ratio are shown in Fig. 4.2. The maximum aspect ratio will determine whether or not the asymptotic technique will become numerically unstable, but the circulation averaged aspect ratio is a better indicator of the overall precision of the method. The new scheme works well under all conditions.

5. A computational demonstration using laboratory experiments of vortex filamentation. Low order vortex methods have been successful in a wide range of scientific and engineering applications. High-order methods are more complex and therefore more difficult to implement in large scale computations. In this section, we present a proof-of-concept calculation to show that the new method is suitable for large-scale computational challenges. The demonstration will consist of the vortex method calculation, a pseudo-spectral method calculation and a physical experiment. More information about a direct comparisons between pseudo-spectral methods and vortex methods can be found in [6]. Similarly, quantitative comparisons between pseudo-spectral and another Lagrangian method called contour surgery have been explored as well [14].

We performed a study of filamentation in a vorticity distribution based on that used in the 1987 paper by Melander, McWilliams and Zabuski [22]. The initial conditions are an elliptical vortex with a smooth transition between rotational and ir- 
rotational fluid. This relatively simple initial condition has continuous derivatives everywhere and a single parameter $\left(\left(R_{o}-R_{i}\right) / R_{o}\right)$ that characterizes the sharpness of the interface. We begin with an axisymmetric vorticity distribution:

$$
\begin{gathered}
g_{\text {axi }}\left(r, R_{i}, R_{o}\right)= \begin{cases}1, & r \leq R_{i}, \\
1-f_{\kappa}\left(\frac{r-R_{i}}{R_{o}-R_{i}}\right), & R_{i}<r<R_{o}, \\
0, & r \geq R_{o},\end{cases} \\
f_{\kappa}(r)=\exp \left[-\frac{\kappa}{r} \exp \left(\frac{1}{r-1}\right)\right], 0 \leq r \leq 1 \\
\kappa=\frac{1}{2} e^{2} \ln (2)
\end{gathered}
$$

The original paper [22] is not specific on how one translates $g_{\text {circ }}$ into an elliptical distribution, but we take the obvious course and define

$$
g\left(x, y, R_{i}, R_{o}, a^{2}\right)=g_{\mathrm{axi}}\left(\sqrt{\frac{x^{2}}{a^{2}}+y^{2} a^{2}}, R_{i}, R_{o}\right),
$$

where $a^{2}$ is the aspect ratio. The specific initial conditions for the proof of concept are

$$
\omega(x, y, 0)=20 g(x, y, 0,1,2)
$$

corresponding specifically to one of the cases studied in [22].

To perform vortex method computations, we discretized (5.3) using $N=11,269$ axisymmetric basis functions with a core size of $\sigma^{2}=3.125 \times 10^{-3}$. The number of basis functions is roughly equivalent to the $128^{2}$ Fourier modes used in [22], but the vortex computation is grid-free and requires no hyperviscosity to damp the artificial growth of high frequency components. We chose a timestep of $10^{-3}$ and used third order Adams-Bashforth to integrate trajectories for the vortex method.

For some flows, the deforming basis functions may continually elongate over time. If unchecked, the deformation will lead to a catastrophic loss of spatial accuracy. We solved this problem using field interpolation. When some or all of the basis functions become extremely deformed, we replace all of them with a collection of axisymmetric basis functions on a regular mesh. The central problem in field interpolation is to develop a stable technique for selecting the circulations of the new basis functions so that they will represent the vorticity field expressed by the original set of deformed basis functions. While there is no viscosity or hyperviscosity introduced into the dynamics of the problem, field interpolation with a fixed core size removes higherfrequency components and thus can be interpreted as a form of low-pass filter. There are a number of ways to perform this type of remeshing including using M-functions $[26,27,28]$ or radial basis functions $[1,2]$. Instead, we use a technique motived by methods use to deblur images.

We use the function $f(\vec{x})$ to represent the vorticity field at some time in the simulation when some or all of the basis functions have become extremely deformed. We will replace all the old basis functions with a new regular mesh of axisymmetric basis functions that induce a field that is controllably close to $f$. The challenge is to choose circulations that accurately represent $f$. We begin by creating a naive 
approximation to $f$ called $f_{1}$ :

$$
\begin{aligned}
f_{1}(\vec{x}) & =\sum_{i=0}^{N} \gamma_{i} \phi\left(\vec{x}-\vec{x}_{i}, \sigma, 1\right) \\
\gamma_{i} & =f\left(\vec{x}_{i}\right) h^{2}
\end{aligned}
$$

where $\vec{x}_{i}$ are the locations of the $i^{\text {th }}$ basis functions on a regular mesh, the $\gamma_{i}$ 's are the circulations of the new basis functions which will replace the existing ones and $h$ is the mesh width. Note that the basis functions are a $\delta$ sequence as $\sigma \rightarrow 0$. Since, $\sigma$ is finite, $f_{1}$ is a blurred representation of $f$. The overlap ratio $\beta=h / \sigma$ is connected to the accuracy of the basis function interpolation. The blurred approximation $f_{1}$ is a Riemann sum for the convolution

$$
f_{1}(\vec{x})=\sum_{i=1}^{N} \frac{f\left(\vec{x}_{i}\right) h^{2}}{4 \pi \sigma^{2}} \exp \left(-\frac{\left|\vec{x}-\vec{x}_{i}\right|^{2}}{4 \sigma^{2}}\right) \approx \iint_{-\infty}^{\infty} \frac{f(\vec{y})}{4 \pi \sigma^{2}} \exp \left(-\frac{|\vec{x}-\vec{y}|^{2}}{4 \sigma^{2}}\right) d y_{1} d y_{2} .
$$

It can be shown that $f_{1}$ is a second order (in $\sigma$ ) approximation to $f$, but we will see that this can be improved considerably.

The Gaussian blurring in (5.6) corresponds to the solution $u\left(\vec{x}, t=\sigma^{2}\right)$ to the PDE

$$
u_{t}=\nabla^{2} u, \quad u(\vec{x}, 0)=f .
$$

In other words, (5.5) is a discrete approximation to the convolution which solves the heat equation (5.7), $f_{1} \approx u\left(\vec{x}, t=\sigma^{2}\right)$. We see that the core size of the basis function $\sigma$ and time $t$ are inextricably linked. The larger the core, the greater the blurring and vice versa. Deblurring is a famously ill-posed problem because high frequencies grow with time. However, we note that our convergent limit is when $\sigma$ is small. We choose smaller $\sigma$ 's for more accurate field interpolation which means we integrate the reverse heat equation for shorter durations to deblur (5.5). There are a number of successful deblurring methods from the image processing community. A detailed review of PDE methods for deblurring is beyond of the scope of this paper, and we refer the reader to sources such as [36] for a more thorough treatment.

We note that our application differs from image enhancement because we seek to reverse a convolution. Methods such as shock filters may help accentuate contours, but do not necessarily serve the purpose of accurately reproducing the vorticity field. Formally reversing the heat equation for an arbitrary fixed time is an ill-posed problem, and explicit numerical methods will amplify high frequency components. However, we are seeking to reverse (5.7) given data at time $t=\sigma^{2}$ where $\sigma$ is our small numerical parameter. If we refine the field interpolation mesh to improve our spatial resolution, we decrease the total integration time for the backward heat equation. Our refinement method is fairly simple.

1. We begin with a vorticity field $f$ that is represented by any set of basis functions. The configuration may be highly disordered or some basis functions might be extremely elongated.

2. We calculate the induced vorticity on a regular grid.

3. We calculate $\gamma^{\prime}$ at each mesh point on the regular grid using (5.5) where $h=\sigma$ is the mesh spacing. The circulations $\gamma^{\prime}$ induce field $f_{1}$.

4. We calculate $\nabla^{2} \gamma^{\prime}$ using sixth order finite differences. 
5. We calculate $\gamma$ at each mesh point by solving the reverse heat equation (solving (5.7) backward in time) using fourth order Runge-Kutta. These $\gamma$ 's are the circulations of a new configuration of axisymmetric basis functions arranged on the regular grid that replaces the original configuration. These deblurred circulations induce a high precision approximation to $f$.

It can be shown that this method yields a sixth order convergence rate for approximating $f$. For detailed analysis and diagnostics of the deblurring method, see [3].

For numerical comparison, we also implemented a pseudo-spectral method. We used the algorithm described in [20], which has been used for the simulation of vortex filamentation in previous studies [15, 22]. The method is based on Fourier expansions of the vorticity and stream functions and solves

$$
\omega_{t}+\psi_{x} \omega_{y}-\psi_{y} \omega_{x}=-\nu_{h} \Delta^{2} \omega, \quad \Delta \psi=-\omega,
$$

where $\nu_{h} \Delta^{2} \omega$ is a hyperviscosity term. The simulation was performed in the doubleperiodic domain with two domains at the same resolution to understand the impact of far field boundary conditions. We performed one calculation on the domain $[-\pi, \pi] \times$ $[-\pi, \pi]$ using a $256 \times 256$ grid and another on the domain $[-2 \pi, 2 \pi] \times[-2 \pi, 2 \pi]$ using a $512 \times 512$ grid. Both used a hyperviscosity $\nu_{h}=10^{-7}$. To advance in time, we used a third order Adams-Bashforth scheme with $\Delta t=0.0005$.

For the problem at hand, both methods performed well and require modest computational resources. Plots of the results obtained from both numerical schemes are presented in Fig. 5.2. Both calculations resolve the position and shape of the filaments and the core and are in agreement with each other. A systematic comparison between both schemes is beyond the scope of this article. As pointed out in [6], the performance of each method is very much dependent on the way boundary conditions are treated and on the driving forces of the fluid motion. While periodic boundary conditions are optimal for spectral methods, since the solution of the Poisson equation is trivial, imposing other restrictions at boundaries substantially increases the cost of these methods. However, for problems on unbounded domains, spectral schemes often require artificial boundary conditions such as absorbing boundary layers [17]. On the other hand, many particle methods, including the one used in this paper, naturally satisfy the boundary conditions on unbounded domains. Another way to address this issue which we did not pursue is to sacrifice spectral accuracy and use finite differences in the radial direction (remapping $[0, \infty)$ onto a finite interval) together with a spectral discretization in the azimuthal direction [5]. For these calculations, the pseudo-spectral calculation with the $[-\pi, \pi]$ smaller domain produced significant overrotation, roughly 17 degrees, in the simulation. This maximum discrepency is reduced to roughly 3 degrees at the end of the 1.65 second time simulation using the $[-2 \pi, 2 \pi]$ larger domain.

The raison d'etre of many large scale scientific computations is to simulate a physical process. To complement our numerical proof-of-concept, we also present a comparison with laboratory experiments on electron vortices, which are trapped electron columns radially contained by an axial magnetic field and axially contained by electric fields [8]. The fast electron motions in the axial direction average over axial variations and the $2 \mathrm{D} \mathbf{E} \times \mathbf{B}$ drift approximation is valid, so to a good approximation the columns evolve according to the 2D Euler equations. To first order, therefore, the electron density distribution evolves as would an equivalent distribution of vorticity in an incompressible and inviscid 2D fluid, contained in a circular tank with a free-slip boundary condition. The isomorphism of the two physical systems is known as the $2 \mathrm{D}$ fluid analogy [4]. 


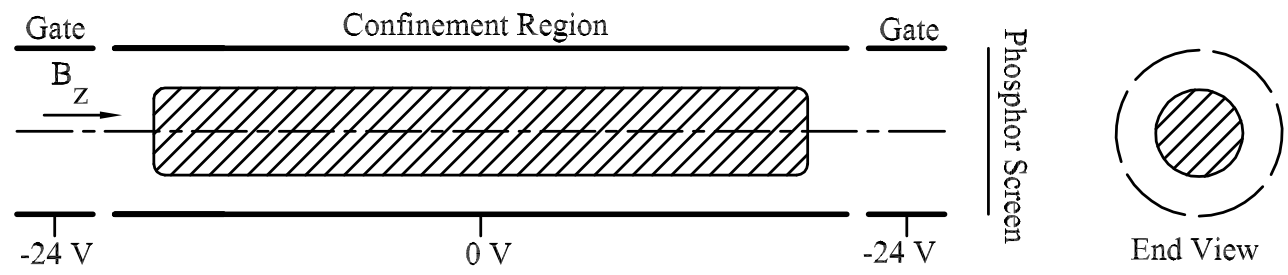

Fig. 5.1. Side- and end-view schematics of the cylindrical electron Malmberg-Penning trap geometry.

There are several advantages to using electron vortices, rather than conventional fluids, to experimentally study vortex dynamics: boundary layers do not form at the wall, dissipation is lower, and electron vorticity is relatively easy to create, manipulate and measure. Electron vortex experiments, buttressed by analytic theory and simulations, have been used to successfully investigate a variety of 2D fluid phenomena, such as vortex merger [10, 23], axisymmetrization from resonant wave-fluid interactions [37], surface mode down-scattering [19], turbulent relaxation [12] and vortex crystal formation [9]. Of course, as with conventional fluid experiments there are aspects of the electron vortex system which fall outside of the 2D fluid analogy. In electron vortex experiments the confinement geometry and the electron parameters are adjusted to eliminate or minimize these non-2D effects, which can include frequency shifts [23], mode instabilities [31, 11] and temperature-dependent transport [30].

A schematic of the experimental apparatus used is shown in Fig. 5.1. A column of electrons of temperature $T \simeq 1 \mathrm{eV}$ is confined inside a series of conducting rings of wall radius $R_{w}=2.88 \mathrm{~cm}$ and confinement length $L_{c}=36.0 \mathrm{~cm}$, in a uniform axial magnetic field $B_{z}=454 \mathrm{Ga}$. This particular trap geometry is often known as a Malmberg-Penning trap [23]. The rings are segmented six-fold into $60^{\circ}$ sectors to allow for vortex manipulation through the application of non-axisymmetric electric fields. The magnetic field provides radial confinement, and negative confinement voltages of $V_{c}=-24 \mathrm{~V}$ applied to end gate rings provide axial confinement. The $x, y$ flow of the electrons is well described by the $2 \mathrm{D}$ drift Poisson equations, with the vorticity of the flow, $\omega \equiv \hat{z} \cdot \nabla \times \mathbf{v}=\left(\frac{4 \pi e c}{B}\right) n=0.399 n$, proportional to the $2 \mathrm{D}$ electron density [4].

In the measurements an electron column is injected and trapped, then made elliptical by the application of precise voltage waveforms onto two opposing ring sectors. To study the subsequent evolution at a specific time, the electron column is dumped axially onto a phosphor screen biased to $9 \mathrm{kV}$, and an image is recorded with a CCD camera of the emitted light, which is proportional to the electron line charge number distribution $N(x, y)$. From the line charge, the electron temperature, and the trap geometry, we calculate the equivalent $2 \mathrm{D}$ density $n(x, y)$ and then the vorticity $\omega(x, y)$ using $\omega=0.399 n$. Because the density measurement is destructive, multiple shots beginning with identical initial conditions are required to follow the time evolution.

We adjusted the electron source and manipulated the injected electron column to create a density initial condition similar to that specified by (5.3). We plot the measured vorticity at three subsequent times in the bottom row of Fig. 5.2. To compare results, we rotate the vorticity fields from the computational experiments by 124.6 degrees so that the first frame of the each time series is horizontal. The strong agreement between laboratory and numerical experiments in this inviscid study demonstrate the value of high-order computations, even with moderate computational resources. This 


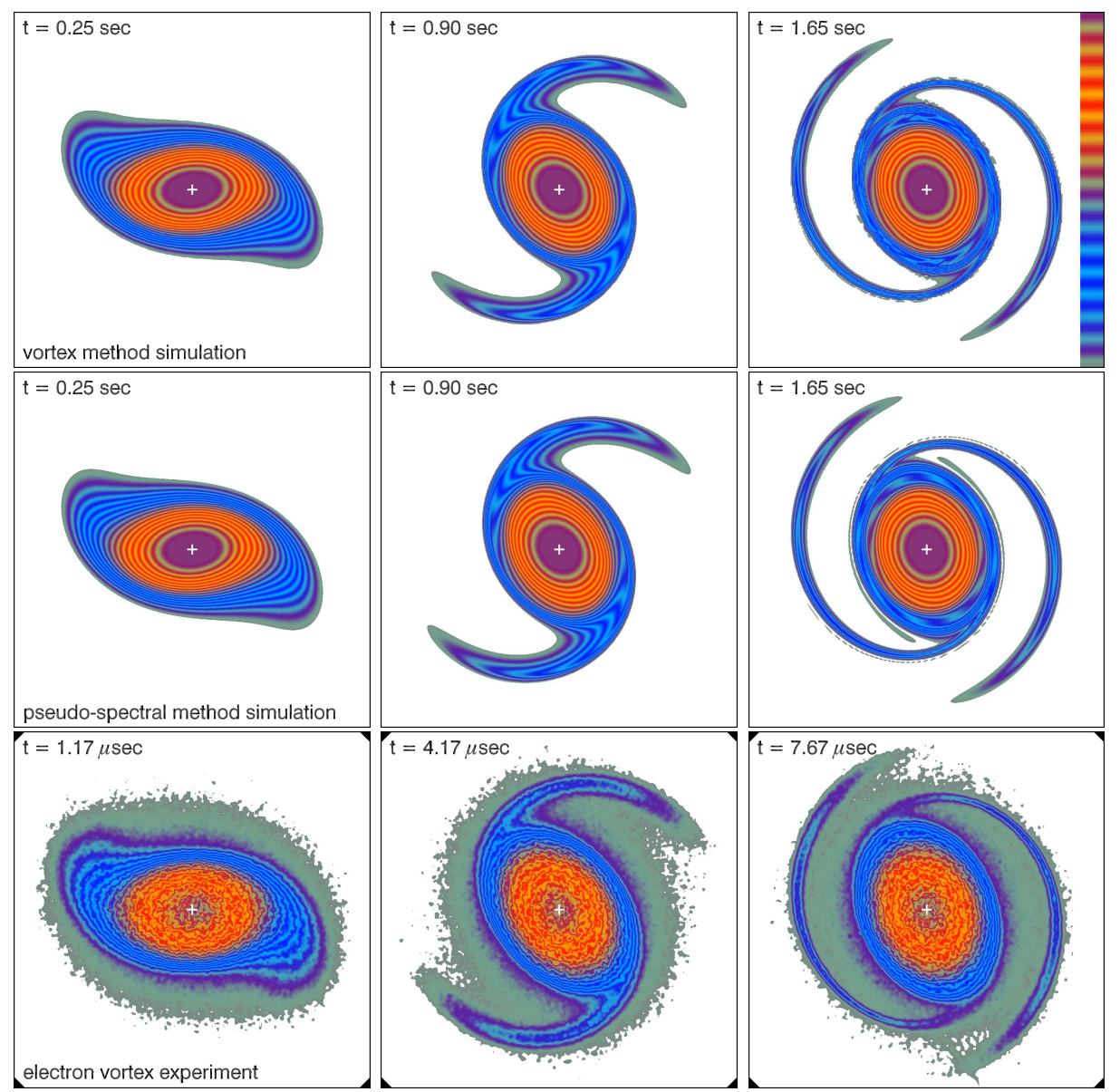

FIG. 5.2. Computational and experimental measurements of vorticity. The top row plots are simulations using the vortex method. The middle row plots are pseudo-spectral computations. The bottom row plots are measurements of an electron vortex. The cylindrical boundary of the experimental trap is indicated in the corners of the experimental plots. The simulation and experiment times are indicated in the plots. Both scales are normalized by the maximum value which is $20 \mathrm{sec}^{-1}$ for the simulations and $4.07 \times 10^{6} \mathrm{sec}^{-1}$ for the experiments.

measurement and others like it are being used to study the axisymmetrization of vortices. This particular example highlights the central role filamentation can play in asymmetrization when the core and filaments interact strongly to reduce the overall aspect ratio [22]. This process is ubiquitous in physical flows and with 2D elliptical vortices is often the dominant axisymmetrization process, although surface mode instabilities [24] and resonant wave-fluid interactions [37, 19] can also play a role.

While the vortex method computation shown in this proof of concept is performed on an infinite domain, we also performed numerical experiments with solid, free-slip boundaries as well. While adding image vortices is an expedient remedy, it can be computationally expensive and is not a solution for general boundary geometries. We implemented a boundary element method that adds a potential flow solution to satisfy the correct boundary conditions on general geometries. Gaussian basis functions do not have finite support, so some vorticity will cross over solid boundaries. However, 


\begin{tabular}{|l|l|l|l|l|}
\hline Technique & Coefficient var's & Precomputation req'd & Accuracy & Performance \\
\hline \hline Asymptotic & $x, y, \epsilon$ or $a$ & No & $\epsilon^{n}$ & Slower \\
\hline Spectral & $a$ & Yes & $\exp (-c n)$ & Faster \\
\hline
\end{tabular}

A summary of features for the two different methods for calculating the Biot-Savart integral. There are implementation, accuracy and performance differences.

Gaussian basis functions decay exponentially so the effect is negligible.

6. Conclusion. In summary, we have presented a new method for approximating the Biot-Savart integral of an elliptical Gaussian basis function. This technique brings about a major improvement in computational efficiency and the range of applicability of high order vortex methods using deforming basis functions. In particular, the new scheme for approximating the streamfunction offers several distinct advantages over the asymptotic scheme which was the only technique available prior to this work. The key differences in implementation, accuracy and performance are summarized in Table 6.1.

The evaluation method performs as well as the asymptotic technique for small or moderate aspect ratios, but for larger aspect ratios, errors accumulate and destroy the extra precision of the high order vortex method when using the asymptotic method. Moreover, the method is faster and since direct evaluations are the dominant part of a vortex computation, even when using fast summation, the new technique boosts the performance of the full vortex method considerably. We have demonstrated the viability of the high order method for large scale computations. While low order axisymmetric methods allow fast, analytic evaluation of the streamfunction, the high order method will always outperform low order methods eventually, and the transition often occurs at moderate problem sizes (see [34] for example). Using a simple remeshing scheme, we have performed a simple vortex filamentation simulation which shows strong agreement with electron vortex experiments and a pseudo-spectral method. In short, the new evaluation technique described in this paper augments and expands the capability of high spatial order vortex methods for viscous flow calculations.

7. Acknowledgements. We performed the sample simulations in this manuscript using the University of Delaware Department of Mathematical Science's Opteron cluster supported by NSF SCREMS DMS-0322583. TBM was supported by National Science Foundation Grant No. PHY-0140318 and U.S. Department of Energy Grant No. DE-FG02-06ER54853. LFR and RBP wish to acknowledge the UD Mathematical Sciences High Performance Computing Roundtable that brought us together to work on this problem.

\section{Appendix A. Overview of the high-order vortex method using elliptical} Gaussian basis functions.

In this section, we formulate the dynamics for a vortex method using elliptical Gaussian basis functions. Generalizing (1.1b), we include parameters for the circulation, position, core size, aspect ratio and orientation of the basis function as follows.

$$
\phi_{\sigma, a, \theta}(\vec{x})=\frac{1}{4 \pi \sigma^{2}} \exp \left(-\frac{\left|A_{\theta, a} \vec{x}\right|^{2}}{4 \sigma^{2}}\right), \quad A_{\theta, a}=\left[\begin{array}{cc}
\cos \theta / a & \sin \theta / a \\
-a \sin \theta & a \cos \theta
\end{array}\right] .
$$

To briefly summarize the systematic derivation in [33], we represent the vorticity field 
as a linear combination of basis functions,

$$
\omega=\sum_{i=1}^{N} \gamma_{i} \phi_{\sigma_{i}, a_{i}, \theta_{i}}\left(\vec{x}-\vec{x}_{i}\right) .
$$

We require that the basis functions satisfy evolution equation

$$
\partial_{t} \phi+(\vec{u}+D \vec{u} \vec{x}) \phi=\frac{1}{\operatorname{Re}} \nabla^{2} \phi
$$

where $\vec{u}$ is a known velocity field determined by the Biot-Savart integral discussed in this paper evaluated at the basis function centroid and $D \vec{u}$, is the matrix of partial derivatives of the velocity field $\vec{u}$.

Inserting (A.1) into (A.3) yields the following equations:

$$
\begin{aligned}
\frac{d}{d t} \vec{x}_{i} & =\vec{u}\left(\vec{x}_{i}\right)+\sigma_{i}^{2}\left(\vec{u}_{x x}\left(\vec{x}_{i}\right) M_{x x}+2 \vec{u}_{x y}\left(\vec{x}_{i}\right) M_{x y}+\vec{u}_{y y}\left(\vec{x}_{i}\right) M_{y y}\right), \\
\frac{d}{d t}\left(\sigma_{i}^{2}\right) & =\frac{1}{2 \operatorname{Re}}\left(a_{i}^{2}+a_{i}^{-2}\right), \\
\frac{d}{d t}\left(a_{i}^{2}\right) & =2\left[d_{11}\left(c_{i}^{2}-s_{i}^{2}\right)+\left(d_{12}+d_{21}\right) s_{i} c_{i}\right] a_{i}^{2}+\frac{1}{2 \sigma^{2} \operatorname{Re}}\left(1-a_{i}^{4}\right), \\
\frac{d}{d t} \theta_{i} & =\frac{d_{21}-d_{12}}{2}+\left[\frac{d_{21}+d_{12}}{2}\left(s_{i}^{2}-c_{i}^{2}\right)+2 d_{11} s_{i} c_{i}\right] \frac{\left(a_{i}^{-2}+a_{i}^{2}\right)}{\left(a_{i}^{-2}-a_{i}^{2}\right)} .
\end{aligned}
$$

Here, $d_{i j}$ are the constituent elements of $D \vec{u}$, the matrix of partial derivatives of the velocity field $\vec{u}, c_{i}=\cos \left(\theta_{i}\right), s_{i}=\sin \left(\theta_{i}\right)$, and the $M$ 's represent velocity field curvatures:

$$
M_{x x}=c_{i}^{2} a_{i}^{2}+s_{i}^{2} / a_{i}^{2}, \quad M_{x y}=c_{i} s_{i}\left(a_{i}^{2}-a_{i}^{-2}\right), \quad M_{y y}=c_{i}^{2} / a_{i}^{2}+s_{i}^{2} a_{i}^{2} .
$$

Basis function deformations are driven by $D \vec{u}$, the local flow deviations. Notice that the velocity field of each basis function is not the velocity field measured at the centroid but rather the velocity field with a curvature correction. This is necessary if one is to achieve fourth order spatial accuracy, so accurate calculation of the velocity field and its derivatives is essential.

\section{REFERENCES}

[1] L. A. Barba. Spectral-like accuracy in space of a meshless vortex method. In ECCOMAS Thematic Conference on Meshless Methods., July 2005.

[2] L. A. Barba, A. Leonard, and C. B. Allen. Advances in viscous vortex methods - meshless spatial adaption based on radial basis functions. Int. J. Num. Methods in Fluids, 47:387421, 2005.

[3] L. A. Barba and L. F. Rossi. Global field interpolation for particle methods. Submitted to $J$. Comput. Phys., 2008.

[4] R. J. Briggs, J. D. Daugherty, and R. H. Levy. Role of Landau damping in crossed-field electron beams and inviscid shear flow. Phys. Fluids, 13:421-432, 1970.

[5] J. D. Buntine and D. I. Pullen. Merger and cancellation of strained vortices. J. Fluid Mech., 205:263-295, 1989.

[6] G.-H. Cottet, B. Michaux, S. Ossia, and G. VanderLinden. A comparison of spectral and vortex methods in three-dimensional incompressible flows. J. Comp. Phys., 175:1-11, 2002.

[7] P. J. Davis. Interpolation and approximation. Dover Publications Inc., New York, 1975.

[8] C. F. Driscoll and K. S. Fine. Experiments on vortex dynamics in pure electron plasmas. Phys. Fluids B, 2:1359-1366, 1990. 
[9] K. S. Fine, A. C. Cass, W. G. Flynn, and C. F. Driscoll. Relaxation of 2 D turbulence to vortex crystals. Phys. Rev. Lett., 75(18):3277-3280, 1995.

[10] K. S. Fine, C. F. Driscoll, J. H. Malmberg, and T. B. Mitchell. Measurements of symmetric vortex merger. Phys. Rev. Lett., 67:588-591, 1991.

[11] J. M. Finn and D. del-Castillo-Negrete. Destabilization of the $m=1$ diocotron mode in non-neutral plasmas. Phys. Rev. Lett., 84(11):2401-2404, 2000.

[12] X.-P. Huang and C. F. Driscoll. Relaxation of 2D turbulence to a metaequilibrium near the minimum enstrophy state. Phys. Rev. Lett., 72:2187-2190, 1994.

[13] H. Lamb. Hydrodynamics, chapter IV, VII, pages 84-86, 232-233. Cambridge University Press, 1993.

[14] B. Legras and D. G. Dritschel. A comparison of the contour surgery and pseudo-spectral methods. J. Comp. Phys., 104:287-302, 1993.

[15] B. Legras, D. G. Dritschel, and P. Caillol. The erosion of a distributed two-dimensional vortex in a background straining flow. J. Fluid Mech., 441:369-398, 2001.

[16] A. Leonard. AIAA 97-0204: Large-eddy simulation of chaotic convection and beyond. In 35th Aerospace Sciences Meeting \& Exhibit. American Institute of Aeronautics and Astronautics, 1997.

[17] A. Mariotti, B. Legras, and D. G. Dritschel. Vortex stripping and the erosion of coherent structures in two-dimensional flows. Physics of Fluids, 6(12):3954-3962, 1994.

[18] J. S. Marshall and J. R. Grant. A method for determining the velocity induced by highly anisotropic vorticity blobs. J. Comp. Phys., 126:286-298, 1996.

[19] N. Mattor, B. T. Chang, and T. B. Mitchell. Beat-wave resonant down scattering of diocotron and Kelvin modes. Phys. Rev. Lett, 96:045003, 2006.

[20] J. C. McWilliams. The emergence of isolated coherent vortices in turbulent flow. J. Fluid Mech., 146:21-43, 1984.

[21] E. Meiburg. Incorporation and test of diffusion and strain effects in the two-dimensional vortex blob technique. J. Comp. Phys., 82:85-93, 1989.

[22] M. V. Melander, J. C. McWilliams, and N. J. Zabusky. Axisymmetrization and vorticitygradient intensification of an isolated two-dimensional vortex through filamentation. $J$. Fluid Mech., 178:137-159, 1987.

[23] T. B. Mitchell and C. F. Driscoll. Electron vortex orbits and merger. Phys. Fluids, 8(7):18281841, 1996.

[24] T. B. Mitchell and L. F. Rossi. The evolution of kirchhoff elliptic vortices. Phys. Fluids, 20:054103, 2008.

[25] P. Moeleker and A. Leonard. Lagrangian methods for the tensor-diffusivity subgrid model. $J$. Comp. Phys., 167:1-21, 2001.

[26] J. J. Monaghan. Why particle methods work. SIAM J. Sci. Stat. Comput., 3(4):422-433, 1982.

[27] J. J. Monaghan. Extrapolating B-splines for interpolation. J. Comp. Phys., 60:253-262, 1985.

[28] J. J. Monaghan. Smoothed particle hydrodynamics. Ann. Rev. Astron. Astrophys., 30:543-574, 1992.

[29] A. Ojima and K. Kamemoto. Numerical simulation of unsteady flow around three dimensional bluff bodies by an advanced vortex method. JSME Int. J. Series B - Fluids and Therm. Mixing, 43(2):127-135, May 2000.

[30] A. J. Peurrung and J. Fajans. A limitation to the analogy between pure electron plasmas and two-dimensional inviscid fluids. Phys. Fluids B, 5:4295-4299, 1993.

[31] S. N. Rasband, R. L. Spencer, and R. R. Vanfleet. Exponential growth of an unstable $l=1$ diocotron mode for a hollow electron column in a warm-fluid model. Phys. Fluids B, 5:669-673, 1993.

[32] G. Riccardi and R. Piva. Motion of an elliptical vortex under rotating strain: conditions for asymmetric merging. Fluid Dyn. Res., 23(2):63-88, 1998.

[33] L. F. Rossi. Achieving high-order convergence rates with deforming basis functions. SIAM J. Sci. Comput., 26(3):885-906, 2005.

[34] L. F. Rossi. A comparative study of Lagrangian methods using axisymmetric and deforming blobs. deforming blobs. SIAM J. Sci. Comput., 27(4):1168-1180, 2006.

[35] L. F. Rossi. Evaluation of the Biot-Savart integral for deformable elliptical gaussian vortex elements. SIAM J. Sci. Comput., 28(4):1509-1532, 2006.

[36] L. I. Rudin, S. Osher, and E. Fatemi. Nonlinear total variation based noise removal algorithms. Physica D, 60:259-268, 1992.

[37] D. A. Schecter, D. H. E. Dubin, A. C. Cass, C. F. Driscoll, I. M. Lansky, and T. M. O'Neil. Inviscid damping of asymmetries on a two-dimensional vortex. Phys. Fluids, 12:2397-2412, 2000.

[38] B. N. Shashikanth and P. K. Newton. Geometric phases for corotating elliptical vortex patches. 
J. Math. Phys., 41(12):8148-8162, 2000.

[39] D. Sipp, D. Fabre, S. Michelin, and L. Jacquin. Stability of a vortex with a heavy core. J. Fluid Mech., 526:67-76, 2005.

[40] Z.-H. Teng. Elliptic-vortex method for incompressible flow at high Reynolds number. J. Comp. Phys., 46:54-68, 1982.

[41] Z.-H. Teng. Variable-elliptical-vortex method for incompressible flow simulation. J. Comp. Math., 4(3):255-262, 1986.

[42] Z.-H. Teng. Convergence of the variable-elliptic-vortex method for Euler equations. SIAM J. Num. Anal., 32(3):754-774, June 1995.

[43] L. N. Trefethen. Spectral Methods in Matlab. Society for Industrial and Applied Mathematics, 2000.

[44] I. Yasuda and G. R. Flierl. Two-dimensional asymmetric vortex merger: merger dynamics and critical merger distance. Dyn. Atmos. Oceans, 26(3):159-181, 1997. 